\title{
Las áreas naturales protegidas y su papel en la conservación de los murciélagos del estado de Tabasco, México
}

\author{
Rodrigo García-Morales ${ }^{1}$, Elías José Gordillo-Chávez ${ }^{2 *}$, Juan de Dios Valdez-Leal² y Coral Jazvel \\ Pacheco-Figueroa ${ }^{2}$
}

Introduction: In the last 50 years Tabasco has changed significantly its original vegetation; most of them were converted to pastureland and croplands. These changes have been threatening biodiversity. In response to this problem, system states of protected natural area was created, with the main goal of the conservation of natural systems and save guard the ecosystem. In fact the knowledge about bats in Tabasco is poor and its importance is not known in protected natural areas. This study is to identify and quantify the richness of bats species in each of the protected natural areas in Tabasco.

Methods: The data of the presence of bat species were obtained through the research of literature studies of bats in different protected areas of the state of Tabasco and fieldwork. The literature research was performed in national and international articles. The field surveys were conducted during the period between January 2006 and December 2010. Every natural protected area was sampled one time by three consecutive nights. The bats capture was done through six mist nets.

Results: We registered six families, 35 gender and 55 bat species in eight natural protected areas. The family Phyllostomidae is the one who had the highest number of species (37) and the frugivore e insectivore were the most diversity trophic guild. We recorded 13 bat species that have some categories of protection in the NOM059-SEMARNAT-2010. The most complementarity sites in natural protected areas were between Parque Estatal Agua Blanca and Reserva Ecológica Río Playa. The state park Agua Blanca and La Sierra are the natural areas with the highest richness in species, this shows the importance of the natural protected area in the conservation of bats.

Discussion and Conclusions: The seven protected natural areas studied harbors $90 \%$ of bat species recorded in the state, most of them in State Parks possibly the most studied ones. Although none is endangered, high percentage are species that live in large continuous forest or forest fragments and are sensitive to changes in coverage of natural vegetation. The diversity of bats reported in this study, is result to the different habitats that are represented in the different protected natural areas. This situation shows the importance of the maintaining of the different habitat types for the conservation of bat diversity in the state. We recommend systematic studies within and outside of protected areas such as inventories, studies on biology and ecology of bats.

Key words: Chiroptera, Complementarity, inventory, Phyllostomidae, State Park

En los últimos 50 años, Tabasco ha experimentado una disminución drástica en su vegetación original, transformándose en pastizales con fines ganaderos y terrenos agrícolas, amenazando de esta manera la biodiversidad del estado. En respuesta a ello se creó en sistema estatal de áreas naturales protegidas de Tabasco con el objetivo de proteger ${ }^{1}$ Centro del Cambio Global y la Sustentabilidad en el Sureste, Calle del Centenario del Instituto Juárez, S/N. Col. Reforma, C.P. 86080. Villahermosa, Tabasco., Tel. +52 (993) 31506112 ext: 115. E-mail: r.garciamorales83@gmail.com (RGM)

${ }^{2}$ División Académica de Ciencias Biológicas, Universidad Juárez Autónoma de Tabasco. Dirección para correspondencia: Km 0.5 Carr. Villahermosa-Cárdenas entronque Bosques de Saloya, Villahermosa 86040, Tabasco, México. E-mail: elias.gordillo@ yahoo.com.mx (EJGC), pachecoral@yahoo.com.mx (CJPF), jdvaldezleal@yahoo.com.mx (JDVL).

${ }^{*}$ Corresponding author 
la biodiversidad y los servicios ecosistémicos que esta nos brinda. Los murciélagos son un grupo abundante y muy diverso, que refleja su diversidad en la variedad de servicios ecosistémicos que desempeña. Actualmente el conocimiento sobre la diversidad de murciélagos en las áreas naturales protegidas de Tabasco es pobre. En este estudio se identifica y cuantifica la riqueza de murciélagos en las diferentes áreas protegidas del estado. Los datos de la presencia de las especies de murciélagos se obtuvieron mediante una búsqueda bibliográfica de estudios realizados con murciélagos en las distintas áreas naturales protegidas del estado, así como de trabajo de campo. Se registró un total de seis familias, 35 géneros y 54 especies de murciélagos. La familia Phyllostomidae fue la que presentó mayor riqueza de especies y los frugívoros e insectívoros fueron los gremios mejor representados. Registramos 13 especies con alguna categoría de protección dentro de la NOM-059-SEMARNAT-2010. La mayor complementariedad entre áreas naturales protegidas se registra entre el Parque Estatal de Agua Blanca y la Reserva Ecológica Río Playa. Recomendamos realizar estudios sistemáticos dentro y fuera de las áreas protegidas, como son inventarios y estudios sobre biología y ecología de los murciélagos.

Palabras clave: Chiroptera, Complementariedad, inventario, Parque Estatal, Phyllostomidae

Introducción

El estado de Tabasco ha sufrido una degradación ambiental drástica. Durante los últimos 50 años ha experimentado políticas de desarrollo que lo llevaron a sustituir la vegetación original (selva perennifolia) por zonas de uso agropecuario. La implementación del Plan Chontalpa (agrícola) y el Plan Balancan-Tenosique (ganadero) desencadenó un acelerado proceso de deforestación que ha provocado la pérdida de más del $95 \%$ de la cobertura vegetal original del estado (Tudela 1990; Zavala y Castillo 2002). Además, la industria petrolera de gran auge a partir de los años 70s en el estado ha provocado un deterioro en la calidad del agua, suelo y aire debido a que los residuos generados no tienen un control y manejo adecuados (Sánchez y Barba 2005).

Debido al desarrollo económico del estado muchas especies de plantas y animales han desaparecido o se encuentran amenazadas. Ante esta situación, la conservación de los recursos naturales del estado adquiere cada vez mayor importancia. Como respuesta a esta necesidad se creó el sistema de áreas naturales protegidas del estado de Tabasco (SANPET) cuyos objetivos son la conservación de los ecosistemas naturales y el mantenimiento de los bienes y servicios que estos ofrecen (SEDESPA 2002). Mediante este instrumento de conservación se han decretado 11 áreas naturales protegidas de jurisdicción estatal y dos federales las cuales protegen alrededor del $15 \%$ del territorio tabasqueño (SEDESPA 2002), buscando con ello garantizar la conservación de ecosistemas con alto valor ecológico, biológico, genético y paisajístico.

Los murciélagos son un grupo abundante y muy diverso, principalmente en las regiones tropicales, donde llegan a representar localmente hasta el $50 \%$ de las especies de mamíferos (Patterson et al. 2003). Su gran diversidad se refleja en la variedad de servicios ecosistémicos que desempeñan. Los murciélagos insectívoros contribuyen al control de las poblaciones de insectos naturales (Kalka et al. 2008), algunos de los cuales pueden 
ser plagas de los cultivos (Kunz et al. 2011); los murciélagos frugívoros son dispersores de semillas de especies pioneras y de vegetación secundaria que favorecen la regeneración de la vegetación después de algún disturbio natural o antropogénico (García-Morales et al. 2012); y las especies nectarívoras son importantes polinizadores de varias plantas con flores, algunas de las cuales son de interés económico para el hombre (Kunz et al. 2011).

En México, la riqueza de murciélagos está constituida por 138 especies, las cuales representan la cuarta parte de los mamíferos del país (Ceballos y Arroyo-Cabrales 2012). En el caso particular del estado de Tabasco se tienen registradas 60 especies (SánchezHernández et al. 2005). A pesar de esta gran diversidad y su valor ecológico se conoce muy poco sobre los patrones de riqueza de este grupo, particularmente se desconoce el papel que juegan las áreas naturales protegidas (ANPs) en la conservación de los murciélagos en Tabasco (Bello-Gutiérrez 2004). El objetivo principal del presente estudio es identificar y cuantificar la riqueza de especies de murciélagos dentro de cada una de las áreas naturales protegidas del estado, mediante la revisión de la literatura disponible y la obtención de datos complementarios en campo. Lo anterior permitirá en primera instancia actualizar el inventario de las especies de murciélagos registradas en cada ANP, además de establecer las bases para elaborar programas de investigación y conservación de este grupo de mamíferos en el estado.

\section{y Métodos}

El estado de Tabasco se localiza en región sureste de México, representando el $1.3 \%$ de la superficie total del país (Figura 1). La fisiografía estatal está conformada por dos provincias: la Llanura Costera del Golfo Sur y la provincia de las Sierras de Chiapas y Guatemala. La primera es de origen aluvial con una altura máxima de $30 \mathrm{~m}$., ocupa el $94 \%$ del territorio estatal, mientras que la segunda ocupa el $6 \%$ restante. El $95.5 \%$ de la superficie del estado presenta un clima cálido húmedo, el restante $4.5 \%$ es clima cálido sub-húmedo hacia la parte este del estado. La temperatura media anual en la entidad es de $27^{\circ} \mathrm{C}$, la temperatura máxima promedio es de $36{ }^{\circ} \mathrm{C}$ y se presenta en el mes de mayo y la temperatura mínima promedio es de $18.5^{\circ} \mathrm{C}$ durante el mes de enero. La precipitación media estatal es de 2,550 $\mathrm{mm}$ anuales, las lluvias se presentan todo el año, siendo más abundantes en los meses de junio a octubre (INEGI 2003). Este clima favorece la presencia de varios tipos de vegetación como selva alta y mediana perennifolia, vegetación ribereña, sabana, manglares, pastizales inducidos y agrosistemas (INEGI 2003). Actualmente la vegetación natural se encuentra fragmentada a consecuencia de las actividades productivas como la ganadería, agricultura y la explotación petrolera (Pérez et al. 2005).

Con respecto a las ANPs, el estado cuenta con 13 reservas que protegen 376,000 hectáreas del estado (Figura 1). El SANPET incluye las categorías de Reserva de la Biosfera (1), Zona de Protección de Flora y Fauna (1) Parque Estatal (2), Reserva Ecológica (6), Parques Ecológicos (1), Monumentos Naturales (1) y Centros de Interpretación y Convivencia con la Naturaleza (1) (Figura 1; SEDESPA 2002).

Revisión de literatura. La obtención de los registros bibliográficos para el estado se realizó mediante una búsqueda intensiva de estudios realizados con murciélagos en las distintas áreas naturales protegidas del estado de Tabasco. Dicha búsqueda se realizó de revistas de circulación nacional e internacional incluidas en la base de datos de JStor, SpringerLink, Science Direct y Google Académico. Sólo se consideraron estudios que fueron realizados 
posteriores a la fecha de declaratoria como área natural protegida, con la finalidad de valorar con mayor objetividad la importancia que cada ANP para la conservación de los murciélagos del estado. Para la búsqueda en internet se incluyeron palabras claves como "mamíferos", "murciélagos", "Tabasco", "inventarios" y "áreas naturales protegidas". Estas palabras fueron escritas en español e inglés. Adicionalmente se consultó la base de datos de la Biblioteca "Dr. Juan José Beauregard Cruz" de la División Académica de Ciencias Biológicas de la Universidad Juárez Autónoma de Tabasco, con la finalidad de obtener información. Los estudios encontrados incluyeron cuatro artículos científicos: Castro-Luna (1999), Guzmán y Bello (2006), Castro-Luna et al. (2007) y García-Morales et al. (2011); dos tesis de licenciatura: De la Cruz (2005) y García-Morales (2008); una tesina: Sosa (2003); y un libro: Sánchez-Hernández y Romero-Almaraz (1995).

Trabajo de campo. Los muestreos de campo se realizaron durante el periodo comprendido entre enero del 2006 y diciembre de 2010. Las áreas naturales protegidas en donde se realizaron los muestreos son: Reserva de la Biosfera Pantanos de Centla, Zona de Protección de Flora y Fauna Cañón del Usumacinta, Parque Estatal la Sierra, Parque Estatal Agua Blanca, Reserva Ecológica Cascadas de Reforma, Río Playa y Yu-Balcah. Cada ANP se visitó en una ocasión con un esfuerzo de muestreo de $540 \mathrm{mts} / \mathrm{red} / \mathrm{hrs}$. Los murciélagos se capturaron en cada sitio mediante el empleo de seis redes de niebla de $12 \mathrm{mts}$ de largo x $2.5 \mathrm{mts}$ de altura, las cuales permanecieron abiertas por un periodo de $5 \mathrm{hrs}$ a partir del anochecer (aproximadamente 18:30 h). La identificación de las especies se realizó mediante la clave de campo de Medellín et al. (2008). De manera general la nomenclatura taxonómica de las especies se basó en la propuesta de Ramírez-Pulido et al. (2014).

Los gremios tróficos fueron determinados a partir de la literatura (Ceballos y Navarro 1991; Kalko y Hadley 2001; Giannini y Kalko 2004). Los datos sobre las categorías de riesgo en las que se encuentran las especies a nivel nacional están basados en la Norma Oficial Mexicana NOM-059-SEMARNAT-2010 (SEMARNAT 2010). Las categorías de cada ANP fueron tomadas del SANPET (2002).

Con la información obtenida se elaboró una base de datos que consistió en los siguientes campos: 1) especie registrada, 2) gremio trófico de acuerdo a la revisión de la literatura para cada una de las especies, 3) categoría de protección de cada especie y 4) categoría del área natural protegida donde se registro la especie. Con esta base de datos se determinó la riqueza y el porcentaje de gremios tróficos de las especies de murciélagos para cada una de las ANPs estudiadas. Para medir la complementariedad en la composición de especies entre las distintas ANPs se calculó el índice de Colwell y Coddington (1994), el cual se expresa en porcentaje y varía desde cero cuando ambos sitios son idénticos hasta 100 cuando ambos sitios son completamente distintos. Finalmente, se determinó el grado de importancia para la conservación de los murciélagos de cada una de las ANPs con base al número de especies registradas que se encuentre dentro de alguna categoría de protección.

De la revisión bibliográfica se obtuvieron siete fuentes que abarcan un periodo de 16 años Resultados (1995-2011) durante los cuales se han registrado 49 especies de murciélagos. Del trabajo de campo se capturaron 30 especies y 391 individuos. De ambas fuentes se obtuvo un total de seis familias, 35 géneros y 55 especies de murciélagos registradas en ocho áreas naturales protegidas del estado de Tabasco, quedando sin evaluar por falta de información 
las ANPs Laguna El Camarón, Laguna de las Ilusiones, La Chontalpa, Laguna La Lima y Centro de interpretación y Convivencia con la Naturaleza YUMKA' (Tabla 1). Las familias mejor representadas en número de especies son Phyllostomidae y Vespertilionidae con 37 y siete especies respectivamente, mientras que las menos representadas son las familias Natalidae y Noctilionidae con una sola especie (Tabla 1). Los géneros más diversos son Pteronotus y Myotis, ambos con cuatro especies (Tabla 1). Las áreas naturales protegidas en donde se llevaron a cabo las investigaciones se encuentran dentro de cinco categorías: Reserva de la Biosfera (Pantanos de Centla), Zona de Protección de Flora y Fauna (Cañón del Usumacinta), Parque Estatal (Agua Blanca y La Sierra), Reserva Ecológica (Cascadas de Reforma, Río Playa y Yu-Balcah) y Monumento Natural (Grutas de Coconá).

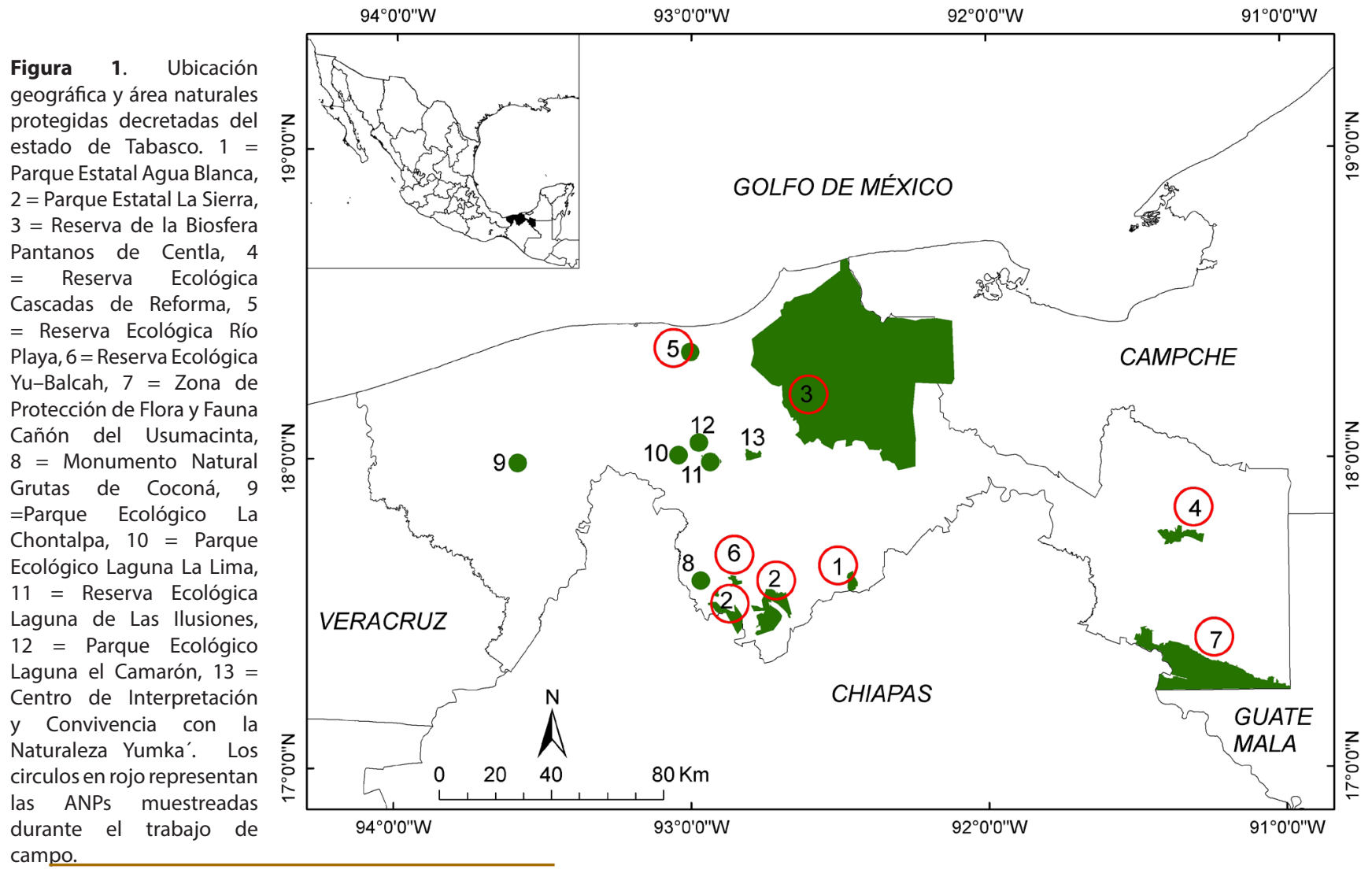

Riqueza y especies con criterio de protección en las áreas naturales protegidas. El Parque Estatal de Agua Blanca y el Parque Estatal La Sierra son las ANPs en donde se tiene la mayor riqueza de especies con 38 y 34 respectivamente, mientras que la Reserva Ecológica Río Playa es el área protegida con el menor número de especies registradas (7; Figura 2). Se encontraron un total de 14 especies de murciélagos con algún criterio de protección de acuerdo con la NOM-059-SEMARNAT-2010 (Figura 2) 10 de las cuales se ubicaron en la categoría de Amenazada (A) y cuatro en la categoría de especie Sujeta a Protección Especial (Pr; Tabla 1). El Parque Estatal de Agua Blanca es el área con el mayor número de especies protegidas (11 especies; Figura 2). La reserva de la Biosfera Pantanos de Centla y el Monumento Natural Grutas de Coconá registraron el menor número de especies protegidas con dos y una especie respectivamente (Figura 2). Del total de especies con algún criterio de protección tres solo se han registrado en el Parque Estatal Agua Blanca (Chrotopterus auritus, Micronycteris schmidtorum y Pteronotus gymnotus) todas ellas con 


\begin{tabular}{|c|c|c|c|c|}
\hline Familia & Especie & Estado de conservación & Gremio trófico & Reserva \\
\hline \multirow[t]{4}{*}{ Emballonuridae } & Balantiopteryx io & & la & 7 \\
\hline & Rhynchonycteris naso & Protección especial & la & 3,4 \\
\hline & Peropteryx macrotis & & la & 2 \\
\hline & Peropteryx kappleri & Protección especial & la & 8 \\
\hline \multirow[t]{5}{*}{ Mormoopidae } & Mormoops megalophylla & & la & 1 \\
\hline & Pteronotus davyi & & la & 1,7 \\
\hline & Pteronotus gymnonotus & Amenazada & la & 1 \\
\hline & Pteronotus parnellii & & la & $1,2,6,7$ \\
\hline & Pteronotus personatus & & la & 1,2 \\
\hline Natalidae & Natalus stramineus & & la & 1,2 \\
\hline Noctilionidae & Noctilio leporinus & & $\mathrm{Pi}$ & $1,3,5$ \\
\hline \multirow[t]{37}{*}{ Phyllostomidae } & Anoura geoffroyi & & $\mathrm{Ne}$ & 2 \\
\hline & Artibeus jamaicensis & & $\mathrm{Fr}$ & $1,2,3,5,6,7$ \\
\hline & Artibeus lituratus & & $\mathrm{Fr}$ & $1,2,3,4,5,6,7$ \\
\hline & Carollia perspicillata & & $\mathrm{Fr}$ & $1,2,4,6,7$ \\
\hline & Carollia sowelli & & $\mathrm{Fr}$ & $1,2,6,7$ \\
\hline & Carollia subrufa & & $\mathrm{Fr}$ & 2 \\
\hline & Centurio senex & & $\mathrm{Fr}$ & $1,2,7$ \\
\hline & Chiroderma salvini & & $\mathrm{Fr}$ & 1,2 \\
\hline & Chiroderma villosum & & $\mathrm{Fr}$ & 1 \\
\hline & Choeroniscus godmani & & $\mathrm{Ne}$ & 2,7 \\
\hline & Chrotopterus auritus & Amenazada & $\mathrm{Ca}$ & 1 \\
\hline & Dermanura phaeotis & & $\mathrm{Fr}$ & $1,2,3,4,6,7$ \\
\hline & Dermanura tolteca & & $\mathrm{Fr}$ & 1,2 \\
\hline & Dermanura watsoni & Protección especial & $\mathrm{Fr}$ & $1,2,4,7$ \\
\hline & Desmodus rotundus & & $\mathrm{He}$ & $1,2,3,7$ \\
\hline & Diphylla ecaudata & & $\mathrm{He}$ & 3 \\
\hline & Glyphonycteris silvestris & & Is & 3 \\
\hline & Glossophaga commissarisi & & $\mathrm{Ne}$ & 2,7 \\
\hline & Glossophaga morenoi & & $\mathrm{Ne}$ & 1 \\
\hline & Glossophaga soricina & & $\mathrm{Ne}$ & $1,2,3,6,7$ \\
\hline & Hylonycteris underwoodi & & $\mathrm{Ne}$ & 1,2 \\
\hline & Lampronycteris brachyotis & Amenazada & Is & 1,7 \\
\hline & Lonchorhina aurita & Amenazada & Is & 1,2 \\
\hline & Lophostoma brasiliense & Amenazada & Is & 1,2 \\
\hline & Lophostoma evotis & Amenazada & Is & $1,2,3$ \\
\hline & Micronycteris microtis & & Is & $1,2,3$ \\
\hline & Micronycteris schmidtorum & Amenazada & Is & 1 \\
\hline & Micronycteris silvestris & & Is & 7 \\
\hline & Mimon cozumelae & Amenazada & Is & $1,2,7$ \\
\hline & Mimon crenulatum & Amenazada & Is & 1,7 \\
\hline & Phyllostomus discolor & & Om & 1 \\
\hline & Platyrrhinus helleri & & $\mathrm{Fr}$ & $1,2,4,5,6,7$ \\
\hline & Sturnira parvidens & & $\mathrm{Fr}$ & $1,2,3,4,5,6,7$ \\
\hline & Sturnira hondurensis & & $\mathrm{Fr}$ & $2,5,6,7$ \\
\hline & Trachops cirrhosus & Amenazada & $\mathrm{Ca}$ & $1,2,4$ \\
\hline & Uroderma bilobatum & & $\mathrm{Fr}$ & $1,2,3,7$ \\
\hline & Vampyressa thyone & & $\mathrm{Fr}$ & $1,2,3$ \\
\hline \multirow[t]{7}{*}{ Vespertilionidae } & Bauerus dubiaquercus & & la & 2 \\
\hline & Eptesicus furinalis & & la & 3 \\
\hline & Myotis californicus & & la & 1 \\
\hline & Myotis carteri & Protección especial & la & 4 \\
\hline & Myotis keaysi & & la & $1,2,4,7$ \\
\hline & Myotis nigricans & & la & 2,7 \\
\hline & Rhogeessa tumida & & la & 5 \\
\hline
\end{tabular}

Tabla 1. Riqueza de especies, gremios tróficos y estado de conservación de las especies del orden Chiroptera presentes en las áreas naturales protegidas del estado de Tabasco, México. El estado de conservación fue de acuerdo a la NOM-059SEMARNAT-2010.

\footnotetext{
$\mathrm{Ca}=$ carnívoro, $\mathrm{Fr}=$ frugívoro, $\mathrm{He}=$ hematófago, la = insectívoro aéreo, Is $=$ insectívoro sustrato, $\mathrm{Ne}=$ nectarívoro, $\mathrm{Om}=$ omnívoro, $\mathrm{Pi}=$ piscívoro. $1=$ Parque Estatal Agua Blanca, $2=$ Parque Estatal La Sierra $3=$ Reserva de la Biósfera Pantanos de Centla, 4 $=$ Reserva Ecológica Cascadas de Reforma, 5 $=$ Reserva Ecológica Río Playa, 6 = Reserva Ecológica Yu-Balcah, 7 = Zona de Protección de Flora y Fauna Cañón del Usumacinta, 8= Monumento Natural Grutas de Coconá.
} 
categoría de Amenazada (A) y solo una en el Monumento Natural Grutas de Coconá (Peropteryx kappleri) en la categoría de Sujeta a Protección Especial.

Figura 2. Número de especies de murciélagos registradas (barras amarillas) y con criterio de protección (barras rojas) para el estado, en total y para cada una de las áreas naturales protegidas de estudio en Tabasco. PEAB = Parque Estatal Agua Blanca, PELS = Parque Estatal La Sierra, RBPC = Reserva de la Biósfera Pantanos de Centla, RECR = Reserva Ecológica Cascadas de Reforma, RERP $=$ Reserva Ecológica Río Playa, REYB = Reserva Ecológica YuBalcah, ZPCU = Zona de Protección de Flora y Fauna Cañón del Usumacinta, MNGC = Monumento Natural Grutas de Coconá.

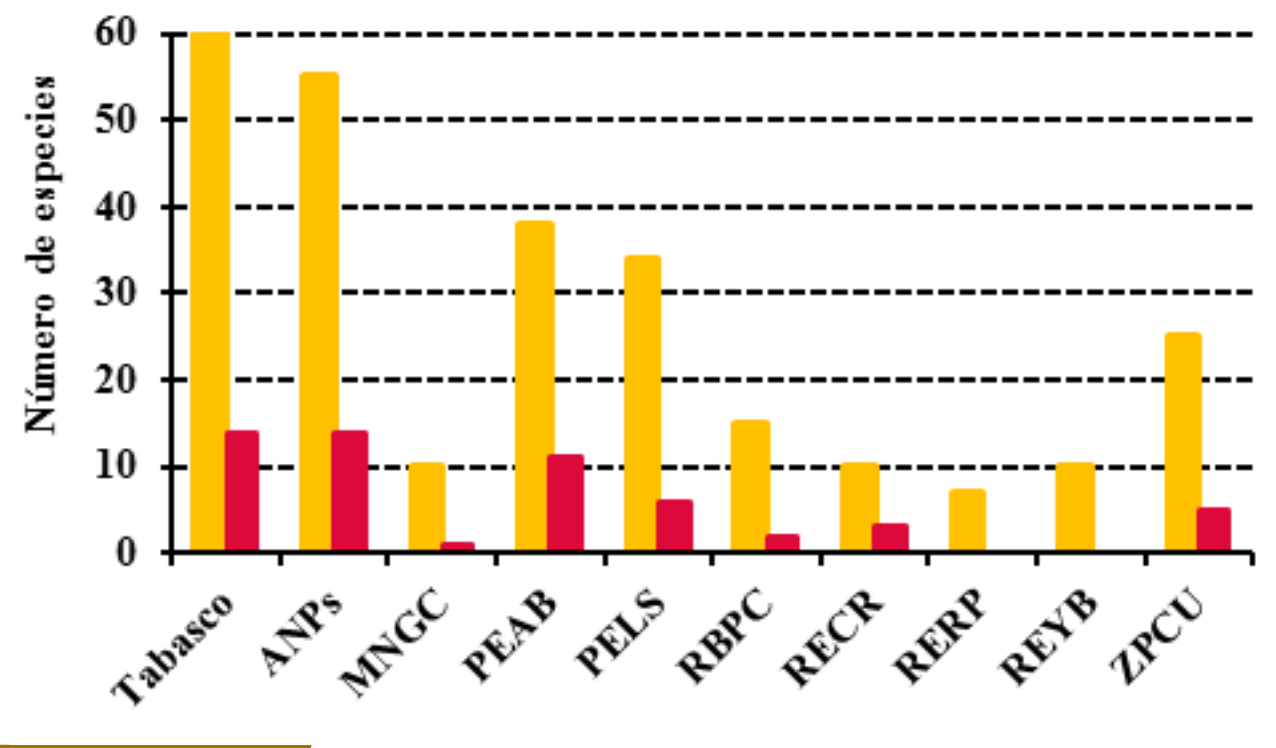

Gremios tróficos. De acuerdo a la forma de alimentación, los gremios insectívoro y frugívoro agrupan el mayor porcentaje de especies con un $49.1 \%$ y $30.9 \%$ respectivamente. Dentro de los insectívoros un $30.1 \%$ son insectívoros aéreos y un $18.9 \%$ son insectívoros de sustrato. Los nectarívoros representan un $9.0 \%$ de las especies, seguido de los gremios hematófago y carnívoro por un $4.0 \%$ y finalmente los omnívoros y piscívoros representados por un $2 \%$ del total de las especies. En el Parque Estatal Agua Blanca se registraron los ocho gremios (Figura 3), mientras que en la Reserva Ecológica de Yu-Balcah solo se registraron dos gremios (Figura 3). En seis de las ocho ANPs evaluadas el gremio frugívoro registra la mayor cantidad de especies. Los gremios menos representados son el omnívoro presente únicamente en el Parque Estatal Agua Blanca y el piscívoro en tres ANPs (Figura 3).

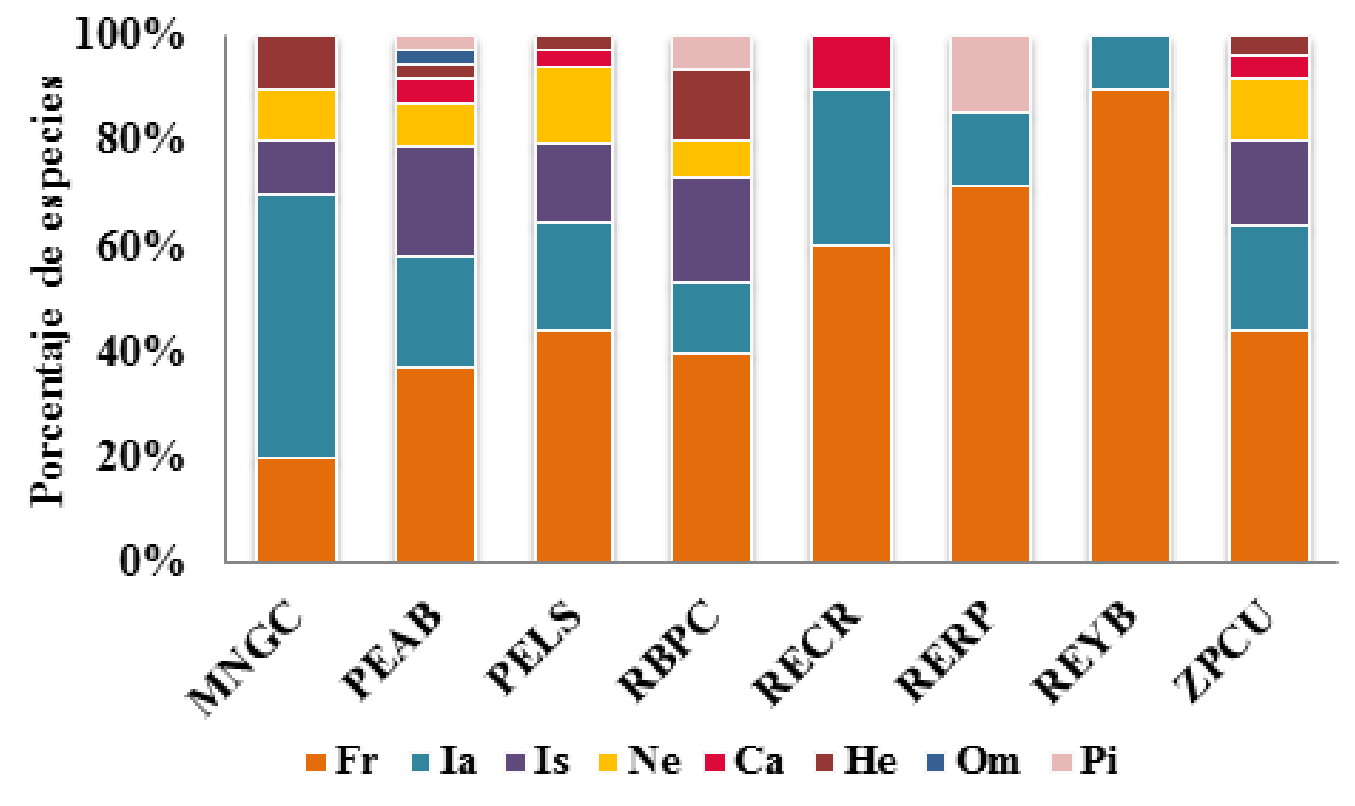

Figura 3. Porcentaje de los diferentes gremios tróficos registrados en cada una de las áreas naturales protegidas estudiadas de Tabasco. PEAB $=$ Parque Estatal Agua Blanca, PELS = Parque Estatal La Sierra, RBPC = Reserva de la Biósfera Pantanos de Centla, $\mathrm{RECR}=$ Reserva Ecológica Cascadas de Reforma, RERP = Reserva Ecológica Río Playa, REYB = Reserva Ecológica Yu-Balcah, ZPCU = Zona de Protección de Flora y Fauna Cañón del Usumacinta MNGC $=$ Monumento Natural Grutas de Coconá. $\mathrm{Ca}=$ carnívoro, $\mathrm{Fr}$ = frugívoro, $\mathrm{He}=$ hematófago, la = insectívor o aéreo, Is = insectívoro sustrato, $\mathrm{Ne}=$ nectarívoro, Om = omnívoro, $\mathrm{Pi}=$ piscívoro. 


\begin{tabular}{lcccccccc}
\hline & MNGC & PEAB & PELS & RBPC & RECR & RERP & REYB & ZPCU \\
\hline MNGC & - & 75 & 77 & 80 & 88 & 93 & 75 & 79 \\
PEAB & $(7)$ & - & 42 & 74 & 79 & 88 & 76 & 56 \\
PELS & $(8)$ & $(26)$ & - & 74 & 78 & 86 & 71 & 53 \\
RBPC & $(4)$ & $(11)$ & $(10)$ & - & 81 & 78 & 75 & 79 \\
RECR & $(2)$ & $(8)$ & $(8)$ & $(4)$ & - & 79 & 67 & 70 \\
RERP & $(1)$ & $(5)$ & $(5)$ & $(4)$ & $(3)$ & - & 58 & 81 \\
REYB & $(4)$ & $(9)$ & $(10)$ & $(5)$ & $(5)$ & $(5)$ & - & 60 \\
ZPCU & $(6)$ & $(19)$ & $(20)$ & $(7)$ & $(8)$ & $(5)$ & $(10)$ & - \\
\hline PEAB = Parque & Estatal Agua Blanca, PELS= Parque Estatal Sierra & RBPC = Reserva
\end{tabular}

Tabla 2. Indice de complementariedad y especies compartidas entre las áreas naturales protegidas de Tabasco.

de la Biosfera Pantanos de Centla, RECR= Reserva Ecológica Cascadas de Reforma $\mathrm{RERP}=$ Reserva Ecológica Río Playa, REYB = Reserva Ecológica Yu-Balcah, ZPCU = Zona de Protección de Flora y Fauna Cañón del Usumacinta. Números entre parentesis = especies compartidas.

Complementariedad entre áreas naturales protegidas. La mayor complementariedad entre áreas naturales protegidas se registra entre el Monumento Natural Grutas Coconá y la Reserva Ecológica Río Playa con un 93.7 \% de disimilitud. La menor completitud en composición de especies son el Parque Estatal de Agua Blanca y Parque Estatal la Sierra (Tabla 2).

El presente trabajo es el primero en compilar la información sobre las especies de murciélagos registradas en las diferentes áreas naturales protegidas de Tabasco. De acuerdo con nuestros resultados el sistema de áreas naturales protegidas del estado de Tabasco, resulta un importante instrumento de conservación para este grupo de mamíferos. Las ocho ANPs estudiadas albergan el $83 \%$ de las familias, $85 \%$ de los géneros y el $90 \%$ de las especies de murciélagos registradas en el estado. Además de la alta riqueza de especies, las ANPs también albergan un porcentaje alto de especies con criterios de protección. De las 38 especies protegidas en México, el 33 \% (13 especies) de ellas se distribuyen en Tabasco (Sánchez-Hernández et al. 2005) y todas ellas han sido registradas en las ANPs, principalmente en los Parques Estatales Agua Blanca y La Sierra. Aunque ninguna está en peligro de extinción, la mayoría de ellas son especies que habitan en selva continua o en grandes fragmentos de selva y son sensibles a cambios en la cobertura de la vegetación natural (Galindo-González 2004; Castro-Luna et al. 2007). Por ello el mantenimiento de grandes extensiones de vegetación natural dentro de las ANPs es vital para la conservación de estas especies considerando que fuera de las ANPs los remanentes de vegetación natural extensos son escasos. En el caso de la Reserva de la Biosfera Pantanos de Centla, el ANP de mayor extensión en el estado, se requieren de más estudios para evaluar su importancia en la conservación de los murciélagos.

La fauna de murciélagos de las ANPs está dominada por la familia Phyllostomidae (74\% de las especies registradas). Esta familia es exclusiva del continente Americano y la de mayor diversidad de especies en las regiones tropicales del continente. Localmente llegan a representan entre el 60 y $70 \%$ de las especies en los ensambles de murciélagos neotropicales (Lim y Engstrom 2005). Se ha demostrado que algunas 
especies de la familia Phyllostomidae se benefician de los procesos de fragmentación e incrementan su abundancia cuando la vegetación natural es modificada (GalindoGonzález 2004). Sin embargo existen otras especies como Centurio senex, Chrotopterus auritus, Mimon cozumelae, M. crenulatum, Trachops cirrosus y Vampyressa thyone que por sus requerimientos de hábitat tan especializados son sensibles a las modificaciones del hábitat (Galindo-González 2004). Estas especies fueron registradas en las distintas ANPs del estado, de ahí su importancia para la conservación de estos murciélagos.

Se ha demostrado que las redes de niebla están sesgadas hacia la captura de especies pertenecientes a la familia Phyllostomidae (Pech-Canché et al. 2011), por lo que ésta suele estar mejor representadas que otras familias en muchos de los estudios realizados en el Neotrópico con este método de captura (Giannini y Kalko 2004). En este trabajo destaca la ausencia de las especies de la familia Molossidae, previamente reportadas para el estado (Sánchez-Hernández et al. 2005). Las especies de la familia Molossidae, suelen estar menos representadas en los estudios que utilizan redes de niebla como método de captura debido a sus hábitos de forrajeo por encima del dosel arbóreo (Pech-Canché et al. 2011).

El gremio trófico dominante es el frugívoro, con el 34 \% de las especies registradas. Este patrón ha sido observado en localidades del centro y sur de América (Patterson et al. 2003). Los murciélagos frugívoros son considerados uno de los grupos más efectivos en la dispersión de semillas en regiones tropicales. Las semillas que dispersan los murciélagos son en su mayoría especies pioneras y características de vegetación secundaria como Solanaceae, Piperaceae y Cecropiaceae (García-Morales et al. 2012). Estas especies son claves en los procesos de regeneración de los bosques después de ocurrido un disturbio, ya que son de rápido crecimiento y alta tolerancia a las condiciones ambientales extremas que presentan los espacios deforestados.

De acuerdo al análisis de complementariedad las zonas más similares son Parque Estatal Agua Blanca y Parque Estatal la Sierra (40.4 \%) mientras que entre el Monumento Natural Grutas de Coconá y la Reserva Ecológica de Río Playa existe una mayor diferencia en la composición de especies. Esta diferencia puede atribuirse a las diferencias en el tipo de vegetación que existe en ambas ANPs y a las diferencias en el esfuerzo de muestro. En el Monumento Natural Grutas de Coconá la vegetación corresponde a selva mediana perennifolia y en la Reserva Ecológica de Río Playa la vegetación es carácterística de humedales (SEDESPA 2002). Por otro lado en el Parque Estatal Agua Blanca se registraron dos estudios sistematizados en años distintos, en la Reserva Ecológica Río Playa sólo se tiene un trabajo de campo preliminar de pocos días de duración. La cercanía geográfica y las semejanzas en el tipo de vegetación que existe entre el Parque Estatal La Sierra y Agua Blanca podría ser una de las causas por la cual estas dos reservas presentan una similitud en la composición de especies en comparación con las demás ANPs (Pineda et al. 2005, Montiel et al. 2006).

Con base en los resultados obtenidos proponemos las siguientes recomendaciones: 1) Incrementar los estudios sobre murciélagos en las demás ANPs decretadas, ya que algunas no cuentan con estudios de esta índole y no se conoce con certeza el papel que tienen en la conservación de los quirópteros en el estado; los estudios deben realizarse bajo un esquema metodológico que permita realizar comparaciones en estudios posteriores. 2) Se recomienda el empleo de otras técnicas de captura de murciélagos como redes 
de dosel y trampas arpas, así como el empleo de equipos de detección de ultrasonidos para caracterizar de manera más eficiente la comunidad de murciélagos en las ANPs en estudios futuros. 3) Es necesario la realización de estudios ecológicos y biológicos, tales como de reproducción, alimentación, conductuales, uso y preferencia de hábitas, tipos de refugios, papel en la trasmisión de enfermedades emergentes, estado de conservación de las especies y su hábitat. 4) Se recomienda la realización de estudios a largo plazo sobre las dinámicas poblacionales de las especies de murciélagos, sobre todo aquellas que se encuentran dentro de algún criterio de protección. 5) Se propone la evaluación de la quirópterofauna en las áreas de interés para la gestión de corredores biológicos establecidos por la CONABIO (CAT-CGCRB 2012) para evaluar la conectividad entre las ANPs y el potencial flujo de individuos entre las diferentes poblaciones de murciélagos.

El presente trabajo está dedicado a la memoria de J. Bello Gutiérrez (1968-2011) quien fuera un precursor en la conservación de los mamíferos del estado de Tabasco e impulsor en la pasión por la investigación en el manejo y conservación de los dos primeros autores del presente artículo, además de un gran amigo y colaborador. Al Proyecto determinación de la conectividad de fragmentos de vegetación identificados como corredores biológicos en el estado de Tabasco. Clave: TAB-2009 C161277 por el financiamiento para el trabajo de campo.

Agradecimientos

Literatura citada

Bello-Gutiérrez, J. 2004. Mamíferos del estado de Tabasco: Diversidad y especies amenazadas. Kuxulkab Revista de Divulgación 9:5-9.

Castro-Luna, A. A. 1999. Composición y abundancia de la comunidad de murciélagos (Mammalia: Chiroptera) del Parque estatal Agua Blanca, Macuspana, Tabasco Universidad y Ciencia 15:57-68.

Castro-Luna, A. A., V. J. Sosa, y G. Castillo-Campos. 2007. Bat diversity and abundance associated with the degrees of secondary succession in a tropical forest mosaic in south-eastern Mexico. Animal Conservation 10:219-228.

Ceballos, G., y D. Navarro. 1991. Diversity and conservation of American Mammals. Pp. 167-198 en Latin American mammalogy: History, Biodiversity and Conservation. (M. A. Mares, y D. J. Schmidly, eds.). University of Oklahoma, Press. Norman, EE.UU.

Colwell, R. K., y J. A. Coddington. 1994. Estimating terrestrial biodiversity through extrapolation. Philosophical Transactions of the Royal Society of London Series B 345:101-118.

De la CRUZ, F. K. H. 2005. Mamíferos en áreas forestales con diferentes historias de incendios en Boca del Cerro, Tenosique, Tabasco. Tesis de Licenciatura. Universidad Juárez Autónoma de Tabasco.

Galindo-González, J. 2004. Clasificación de los murciélagos de la región de los Tuxtlas, Veracruz, respecto a su respuesta a la fragmentación del hábitat. Acta Zoológica Mexicana 20:239-243.

García-Morales, R. 2008. Diversidad alfa, beta y gamma de murciélagos en el Parque Estatal la Sierra, Tabasco. Tesis de licenciatura. Universidad Juárez Autónoma de Tabasco. Villaermosa, México. 
García-Morales, R., C.E. , y J. Bello Gutiérrez. 2011. Renovando las medidas para evaluar la diversidad en comunidades ecológicas: el número de especies efectivas de murciélagos en el sureste de Tabasco, México. Therya 2:205-215.

García-Morales, R., L. Chapa-Vargas., J. Galindo-González, y E.I. Badano. 2012. Seed dispersal among three different vegetation communities in the Huasteca region, Mexico, analyzed from bat feces. Acta Chiropterologica 14:357-367.

Gianninı, N.P., y K.V. Kalko. 2004. Trophic structure in a large assemblage of phyllostomid bats in Panama. Oikos 105:209-220.

Guzmán Aguirre, C. C., y J. Bello-Gutiérrez. 2006. Mamíferos de Boca del Cerro Tenosique, Tabasco, México. Kuxulkab Revista de Divulgación 11:75-84.

INEGI (Instituto Nacional de Estadistica y Geografia). 2003. Síntesis de información geográfica del estado de Tabasco, anexo cartográfico. Editorial Instituto Nacional Estadística, Geografía e Informática. Ciudad de México, México.

Kalka, M. B., A. R. Smith, y E. K. V. Kalko. 2008. Bats limits arthropods and herbivory in a tropical forest. Science 320:71.

Kalko, E., y C. O. Handley JR. 2001. Neotropical bats in the canopy: diversity, community structure, and implications for conservation. Plant Ecology 153: 319-333.

Kunz, T. H., E. Braun de Torrez, D. Bauer, T. Lobova, y T. H. Fleming. 2011. Ecosystem services provided by bats. Annals of the New York Academy of Sciences 1223:1-38

Lim, B. K., Y M. D. Engstrom. 2005. Mammals of Iwokrama forest. Proceedings of the Academy of Natural Science of Philadelphia 154:71-108.

Medellín, R., H. T. Arita, y O. Sánchez. 2008. Identificación de los murciélagos de México, Clave de campo, 2da edición. Instituto de Ecología Universidad Nacional Autónoma de México. Ciudad de México, México.

Montiel, S., A. Estrada., y P. León. 2006. Bat assemblajes in a naturally fragmented ecosystem in the Yucatan Peninsula, México: species richness, diversity and spatiotemporal dynaimcs. Journal of tropical Ecology, 22:267-276.

Patterson, B. D., M. R. Willig, y R. D. Stevens. 2003. Trophic strategies, niche partitioning, and patterns of ecological organization. Pp. 536-579 en Ecology of bats (Kunz T. H., y M. B. Fenton, eds.). University of Chicago. Chicago, EE.UU.

Pech-Canche, J. M., E. Estrella., D. L. López-Castillo., S. F. Hernández-Betancourt, y C. E. Moreno. 2011. Complementarity and efficiency of bat capture methods in a lowland tropical dry forest of Yucatán, Mexico. Revista Mexicana de Biodiversidad 82:896-903.

Pérez, L.A., M. Sousa., A.M. Hanan., F. Chiang, y P. Tenorio. 2005. Vegetación Terrestre. Pp. 65-110 en Biodiversidad del Estado de Tabasco (J. Bueno., F. Álvarez, y S. Santiago eds.). Instituto de Biología, Universidad Nacional Autónoma de México-Comisión Nacional para el Conocimiento y Uso de la Biodiversidad. Ciudad de México, México.

Pineda, E., C. Moreno., F. Escobar, y G. Halfter. 2005. Frog, Bat and dung beetle diversity in the cloud forest and coffe agroecosystems of Veracruz, México. Conservation Biology 19:400-410.

Ramírez-Pulido, J., J. Arroyo-Cabrales, y A. Castro-Campillo. 2005. Estado actual y relación nomenclatural de los mamíferos terrestres de México. Acta Zoológica Mexicana ( $\mathrm{n}$. s.) $21: 21-85$. 
Ramirez-Pulido, J., N. Gonzalez-Ruíz., A.L Gardner., y J. Arroyo-Cabrales. 2014. List of recent land mammals of Mexico, 2014. Special Publications of the Museum of Texas Tech University 63:1-69.

Sánchez, A. J., y E. Barba. 2005. Biodiversidad de Tabasco. Pp. 1-16 en Biodiversidad del Estado de Tabasco (Bueno. J., F. Álvarez, y S. Santiago eds.). Instituto de Biología, Universidad Nacional Autónoma de México-Comisión Nacional para el Conocimiento y Uso de la Biodiversidad. Ciudad de México, México.

Sánchez-Hernandez, C., y M. de L. Romero-Almaraz. 1995. Murciélagos de Tabasco y Campeche una propuesta para su conservación. Instituto de Biología. Universidad Nacional Autónoma de México. Ciudad de México, México.

Sánchez-Hernández, C., M. de L. Romero-Almaraz, y C. García Estrada. 2005. Mamíferos. Pp. 283-304 en Biodiversidad del Estado de Tabasco (Bueno. J. , F. Álvarez, y S. Santiago eds.). Instituto de Biología, Universidad Nacional Autónoma de México- Comisión Nacional para el Conocimiento y Uso de la Biodiversidad. Ciudad de México, México.

SEDESPA (Secretaría de Desarrollo Social y Protección del Medio Ambiente). 2002. Áreas naturales protegidas del Estado de Tabasco. SEDESPA y Gobierno del Estado de Tabasco. Villahermosa, México.

SEMARNAT (Secretaría de Medio Ambiente y Recursos Naturales). 2010. Norma Oficial Mexicana NOM-059-SEMARNAT-2010, Protección ambiental-Especies nativas de México de flora y fauna silvestres-Categorías de riesgo y especificaciones para su inclusión, exclusión o cambio-Lista de especies en riesgo. Diario Oficial de la Federación, 30 de diciembre de 2010.

Sosa, L. M. A. 2003. Estudio preliminar de la Quiróptero fauna de los Tíntales de reserva de la Biosfera Pantanos de Centla, Tabasco, México: "Estructura y Hábitos alimentarios". Tesis Profesional. Licenciatura en Biología. División Académica de Ciencias Biológicas. Universidad Juárez Autónoma de Tabasco. Villahermosa, México

Tudela, F. 1990. Recursos naturales y sociedad en el trópico húmedo. Pp. 149-182 en Medio ambiente y desarrollo en México (Leff, E., ed.). Universidad Nacional Autónoma de México. Ciudad de México, México.

Zavala, C. J., y O. Castillo. 2002. Cambios de uso de la tierra en el estado de Tabasco. Pp. 38-56 en Plan de uso sustentable de los suelos del Estado de Tabasco (Palma-López, D. J., y A. Triano, eds.). Colegio de Posgraduados. Villahermosa, México.

Sometido: 5 de septiembre de 2014

Revisado: 5 de noviembre de 2014

Aceptado: 30 de noviembre de 2014

Editor asociado: Cristina Macswiney 